\title{
Linear Polynomial-Time Algorithms To Construct 4-Connected 4-Regular Locally Connected Claw-Free Graphs ${ }^{\star}$
}

\author{
MingChu $\mathrm{Li}^{1}$, Liming Xiong ${ }^{2}$, and Hong $\mathrm{Liu}^{3}$ \\ ${ }^{1}$ College of Science, Chongqing Technology and Business University, \\ Chongqing, P.R. China \\ School of Software, Dalian University of Technology \\ Dalian 116620, China \\ ${ }^{2}$ Department of Mathematics, Beijing Institute of Technology \\ Beijing 100081, P.R. China \\ ${ }^{3}$ School of Software, Dalian University of Technology \\ Dalian 116620, China
}

\begin{abstract}
A vertex of a graph is locally connected if its neighborhood is connected. A graph $G$ is locally connected if every vertex of $G$ is locally connected. A graph is called claw-free if it does not contain a copy of $K_{1,3}$ as an induced subgraph. In this paper, we provide a constructive characterization of 4-connected 4-regular locally connected claw-free graphs. From its proof, we can give a linear polynomial-time algorithm to construct a 4-connected 4-regular locally connected claw-free graph.
\end{abstract}

Keywords: constructive characterization, linear polynomial-time, clawfree graph.

\section{Terminology and Notation}

We will consider the class of undirected finite graphs without loops or multiple edges, and use [1] for terminology and notation not defined here. Let $G=(V, E)$ be a graph. For a subgraph $H$ of a graph $G$ and a subset $S$ of $V(G)$, we denote by $G-H$ and $G[S]$ the induced subgraphs of $G$ by $V(G)-V(H)$ and $S$, respectively. A clique of $G$ is a complete subgraph of $G$. A clique with $k$ vertices is called a $k$-clique. We say that a vertex is adjacent to a clique if it is adjacent to at least one vertex of the clique. A subgraph $K$ and a subgraph $B$ are disjoint if the vertices of $K$ and the vertices of $B$ are disjoint. For any vertex $v, N(v)$ denotes the neighborhood of $v$. For $S$ in $V$, let $N(S)=\cup_{x \in S} N(x)$, and $\bar{N}(S)=$ $N(S)-S=\{x \in N(S)$ but $x \notin S\}$. A vertex $v$ of $G$ is locally connected if its neighborhood $N(v)$ is connected otherwise $v$ is locally disconnected. A graph $G$ is locally connected if every vertex of $G$ is locally connected. A graph is called claw-free if it does not contain a copy of $K_{1,3}$ as an induced subgraph. Whenever

^ Supported by Nature Science Foundation of China under grant numbers: 60673046 (M. Li) and 10671014 (L. Xiong). 
$\left\{v, v_{1}, v_{2}, v_{3}\right\}$ denotes an induced claw, then the leftmost vertex $v$ is meant to be the center of the claw.

\section{Results}

There are a lot of results on algorithms and their complexity analysis (see 2], [3, 4], 5] ). In this paper, we will provide a constructive characterization of 4-connected, 4-regular, locally connected, claw-free graphs. From its proof, we can give a linear polynomial-time algorithm to construct a 4-connected 4-regular locally connected claw-free graph. In order to explore our main results, we first prove the following result.

Lemma 1. Let $G$ be a 4-connected 4-regular claw-free graph on $n$ vertices. If $G$ contains a 4-clique, then every vertex of $G$ is contained in a 4-clique, and all 4-cliques are disjoint if $n \geq 6$.

Proof. Let $K$ be a clique in $G$. If there is a vertex in $G$ such that it is not contained in any 4-clique, then there is at least one vertex $w$ in $G$ adjacent to some clique such that $w$ is not contained in any 4-clique. Without loss of generality assume that $K$ is such a 4 -clique adjacent to $w$. Let $V(K)=\left\{v_{1}, v_{2}, v_{3}, v_{4}\right\}$ and $w v_{1} \in E(G)$. Then $N\left(v_{1}\right)=\left\{v_{2}, v_{3}, v_{4}, w\right\}$. Let $w^{\prime}$ be a neighbor of $w$ and $w^{\prime} \neq v_{1}$. If $w^{\prime} \in\left\{v_{2}, v_{3}, v_{4}\right\}$ (say $w^{\prime}=v_{2}$ ), then $N\left(v_{2}\right)=\left\{v_{1}, v_{3}, v_{4}, w^{\prime}\right\}$. If $n=5$, then $G$ is $K_{5}$ and so we are done. So $n \geq 6$. It follows that $G-\left\{v_{3}, v_{4}, w\right\}$ is not connected. Thus $G$ is not 4 -connected. This contradiction shows that $w^{\prime} \notin\left\{v_{2}, v_{3}, v_{4}\right\}$ and $\left(N(w)-\left\{v_{1}\right\}\right) \cap\left\{v_{2}, v_{3}, v_{4}\right\}=\emptyset$. For any two vertices $w_{1}, w_{2}$ in $N(w)-\left\{v_{1}\right\}$, we have $w_{1} w_{2} \in E(G)$ since $G\left[w, v_{1}, w_{1}, w_{2}\right] \neq K_{1,3}$ and $d\left(v_{1}\right)=4$. It follows that $G\left[(N(w) \cup\{w\})-\left\{v_{1}\right\}\right]$ is a 4-clique. This contradiction shows that every vertex of $G$ is contained in a 4-clique. Since $G$ is 4-regular, all 4-cliques are disjoint. So the proof of Lemma 1 is completed.

Lemma 2. Let $G$ be a 4-connected 4-regular claw-free graph on $n \geq 7$ vertices and $v$ a vertex of $G$. If $v$ is locally connected, then $G[N(v)]$ is an induced hamiltonian path in $G[N(v)]$.

Proof. Let $v$ be a vertex in $G$ and $N(v)=\left\{v_{1}, v_{2}, v_{3}, v_{4}\right\}$ such that $G[N(v)]$ is connected. If $G$ contains a 4 -clique, then, by Lemma 1 , every vertex of $G$ is contained in a 4-clique $K$. Assume that $v \in V(K)$ and $G\left[(N(v) \cup\{v\})-\left\{v_{4}\right\}\right]$ is a 4 -clique, then $v_{4}$ is adjacent to at least one vertex of $\left\{v_{1}, v_{2}, v_{3}\right\}$ since $G[N(v)]$ is connected. Let $v_{4} v_{1} \in E(G)$. Then $N\left(v_{1}\right)=\left\{v, v_{2}, v_{3}, v_{4}\right\}$ and $G-\left\{v_{4}, v_{3}, v_{2}\right\}$ is not connected. Thus $G$ is not 4 -connected. This contradiction shows that $G$ does not contain a 4 -clique. It follows that $G\left[N(v)-\left\{v_{i}\right\}\right]$ is not a clique for $i=1,2,3,4$. Assume that $v_{1} v_{2} \notin E(G)$. Since $G[N(v)]$ is connected, there is a path $P$ with three or four vertices connecting $v_{1}$ and $v_{2}$ in $G[N(v)]$. Thus we consider two cases to prove that $G[N(v)]$ is an induced path on 4 vertices with internal vertices of degree 2 and two end-vertices of degree 1 in $G[N(v)]$. 
Case 1. $|V(P)|=3$.

Assume that $V(P)=\left\{v_{1}, v_{3}, v_{2}\right\}$. Since $G\left[v, v_{1}, v_{2}, v_{4}\right\} \neq K_{1,3}, v_{4} v_{1} \in E(G)$ or $v_{4} v_{2} \in E(G)$ (say $v_{4} v_{2} \in E(G)$ ). Note that $v_{3} v_{4} \notin E(G)$ since otherwise $G\left[v, v_{2}, v_{3}, v_{4}\right]$ is a 4 -clique. We have that $v_{4} v_{1} \notin E(G)$ since otherwise $G\left[v_{1}, v_{4}, v_{3}, v_{1}^{\prime}\right] \neq K_{1,3}, v_{1}^{\prime} v_{3} \in E(G)$ or $v_{1}^{\prime} v_{4} \in E(G)$, where $v_{1}^{\prime} \in N\left(v_{1}\right)$ $\left\{v_{3}, v_{4}, v\right\}$. Let $A=\left\{v_{1}^{\prime}, v_{2}, w^{\prime}\right\}$, where $w^{\prime}=v_{3}$ if $v_{4} v_{1}^{\prime} \in E(G)$ and $w^{\prime}=v_{4}$ if $v_{3} v_{1}^{\prime} \in E(G)$. It easy to see that $G-A$ is not connected since $n \geq 7$. Thus $G$ is not 4-connected. This contradiction shows $v_{4} v_{1} \notin E(G)$. Thus $G[N(v)]$ is just an induced hamiltonian path $P=v_{1} v_{3} v_{2} v_{4}$ with internal vertices of 2 degree and two end-vertices of degree 1 in $G[N(v)]$.

Case 2. $|V(P)|=4$.

Assume that $P=v_{1} v_{3} v_{4} v_{2}$. Then $v_{1} v_{4}, v_{3} v_{2} \notin E(G)$ since otherwise, say $v_{1} v_{4} \in$ $E(G), G-\left\{v_{1}, v_{2}, v_{3}\right\}$ is not connected since $\bar{N}\left(\left\{v, v_{4}\right\}\right)=\left\{v_{1}, v_{2}, v_{3}\right\}$, a contradiction. Thus $G[N(v)]$ is just an induced hamiltonian path $P=v_{1} v_{3} v_{4} v_{2}$ with internal vertices of 2 degree and two end-vertices of degree 1 in $G[N(v)]$. It follows that the proof of Lemma 2 is completed.

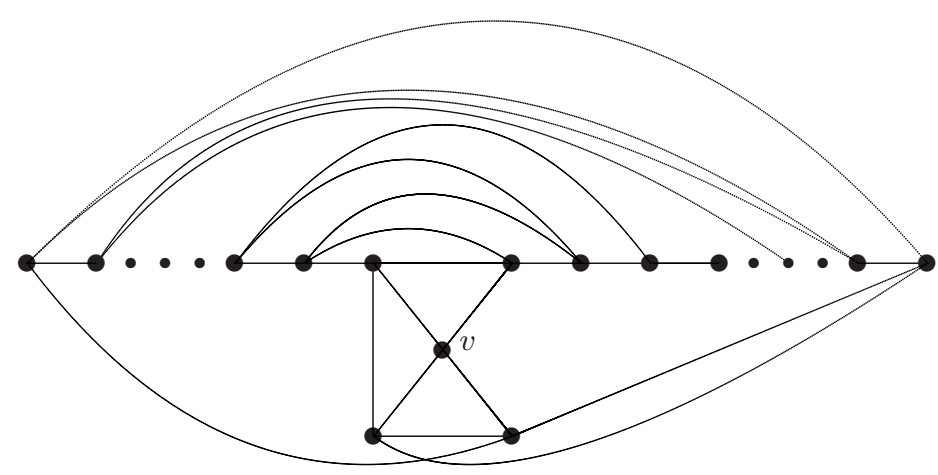

Fig. 1.

Theorem 3. Every 4-connected 4-regular locally connected claw-free graph $G$ on at least 7 vertices is isomorphic to a graph of the depiction in Figure 1.

Proof. Assume that $G$ is not isomorphic to a graph of the form in Figure 1 , and $v$ is any vertex of $G$. Then $v$ is locally connected. By Lemma 2, $N(v)$ is just an induced hamiltonian path in $G[N(v)]$. We construct the graph starting from $v$. Let $N(v)=\left\{v_{1}, v_{2}, u_{1}, u_{2}\right\}$ and $G[N(v)]=v_{2} v_{1} u_{1} u_{2}$. Let $W_{1}=$ $\left\{v_{1}, v_{2}\right\}$ and $W_{2}=\left\{u_{1}, u_{2}\right\}$ and let $W$ be the closed neighborhood of $v$ (i.e., $\left.W=W_{1} \cup W_{2} \cup\{v\}\right)$ and $H=G[W]$. Note that $v_{2} u_{1}, v_{1} u_{2}, v_{2} u_{2} \notin E(G)$. Then 
$N\left(v_{1}\right) \cap N\left(u_{1}\right)=\{v\}$ since otherwise, let $u \in N\left(v_{1}\right) \cap N\left(u_{1}\right)-\{v\}$. Then $\bar{N}\left(\left\{v_{1}, u_{1}, v\right\}\right)=\left\{u, v_{2}, u_{2}\right\}$. Thus $\left\{u, v_{2}, u_{2}\right\}$ is a cut set of $G$ with three vertices, a contradiction. Let $N\left(v_{1}\right)=\left\{v_{2}, v, u_{1}, v_{3}\right\}$. Then $v_{3} u_{1} \notin E(G)$. Adding $v_{3}$ to $W_{1}$. Since $|V(G)| \geq 7$ but $|W|=6, V(G) \neq W$. We have from Lemma 2 that $v_{3} v_{2} \in E(G)$. We further have the following fact.

Claim 1. $v_{3} \notin N\left(W_{2}\right)$.

Recall that $v_{3} u_{1} \notin E(G)$. Now we prove $v_{3} u_{2} \notin E(G)$. If $v_{3} u_{2} \in E(G)$, let $N\left(v_{2}\right)=\left\{v, v_{1}, v_{3}, v_{4}\right\}$. Then we have from $G\left[v_{2}, v_{4}, v, v_{3}\right] \neq K_{1,3}$ that $v_{4} v_{3} \in$ $E(G)$. Since $G\left[v_{3}, v_{4}, v_{1}, u_{2}\right] \neq K_{1,3}$ and $v_{1} v_{4}, v_{1} u_{2} \notin E(G), u_{2} v_{4} \in E(G)$. If $V(G) \neq W \cup\left\{v_{4}\right\}$, then $\left\{v_{4}, u_{1}\right\}$ is a cut-set of two vertices since $v_{1}, v_{2}, v_{3}, u_{2}$ are of degree 4 in $G\left[W \cup\left\{v_{4}\right\}\right]$, a contradiction. Thus $V(G)=W \cup\left\{v_{4}\right\}$ and add $v_{4}$ into $W_{1}$, it follows that $u_{1} v_{4} \in E(G)$ since only $v_{1}, u_{1}, v_{4}$ are of degree 3 in $G[W]$. So $G$ is isomorphic to a graph of the depiction in Figure 1, a contradiction. Thus Claim 1 is true.

Note that $v_{2}$ is of degree 3 and $v_{3}$ is of degree 2 and $v_{1}$ is of degree 4 in $H$. Consider the vertex $v_{3}$ of degree 3 in $H$. By Claim 1, we have $V(G) \neq W$. Let $N\left(v_{2}\right)=\left\{v, v_{1}, v_{3}, v_{4}\right\}$ and add $v_{4}$ into $W_{1}$. From Lemma 2, $v_{i} v_{i-1} \in E(G)$, where $i=4$. We further have the following fact.

Claim 2. If $V(G) \neq W$, then $v_{4} \notin N\left(W_{2}\right)$.

If $v_{4} u_{2} \in E(G)$, let $N\left(v_{3}\right)=\left\{v_{1}, v_{2}, v_{4}, v_{5}\right\}$. Then $v_{5} v_{4} \in E(G)$ since $G\left[v_{3}, v_{5}, v_{4}\right.$, $\left.v_{1}\right\} \neq K_{1,3}$. Since $G\left[v_{4}, v_{2}, v_{4}, u_{2}\right] \neq K_{1,3}$ and $v_{2} v_{5}, v_{2} u_{2} \notin E(G), v_{5} u_{2} \in E(G)$. Thus $d_{H}\left(v_{4}\right)=4$. If $V(G) \neq W \cup\left\{v_{5}\right\}$, then $\left\{v_{5}, u_{1}\right\}$ is a cut set of two vertices, a contradiction. Thus $V(G)=W \cup\left\{v_{5}\right\}$, and add $v_{5}$ into $W_{1}$. Since $d_{H}\left(v_{5}\right)=d_{H}\left(u_{1}\right)=3, u_{1} v_{5} \in E(G)$. Thus $G$ is isomorphic to a graph of the depiction in Figure 1, a contradiction. So $v_{4} u_{2} \notin E(G)$. If $v_{4} u_{1} \in E(G)$, then $G\left[u_{1}, u_{2}, v_{1}, v_{4}\right]=K_{1,3}$. This contradiction shows that Claim 2 is true.

From the above, we have established the following claim.

Claim 3. $v_{i}$ is of degree $2, v_{i-1}$ is of degree 3 , and $v_{j}$ is of degree 4 in $H$ for $j=1,2, \ldots, i-2$, where $i=4$.

Now we return to the proof of Theorem 3. If $V(G)=W$, then we have from $d_{H}\left(u_{2}\right)=2$ that $u_{2} v_{4}, u_{2} v_{3} \in E(G)$. Thus $u_{1} v_{4} \in E(G)$. It follows that $G$ is isomorphic to Figure 1. We assume that $V(G) \neq W$. Then, by Claim 3, consider the vertex $v_{3}$ of degree 3 in $H$ and add the neighbor $v_{5}$ of $v_{4}$ outside $W$ into $W_{1}$. By Lemma $2, v_{5} v_{4} \in E(G)$. Then $v_{5}$ has similar properties in Claim 2 and Claim $3(i=5)$. But $G$ is finite, we must have $V(G)=W$ and $W_{1}=\left\{v_{1}, v_{2}, \ldots, v_{n-3}\right\}$. And Claim 3 is true for $i=n-3$. It follows that $G$ is isomorphic to a graph of the depiction in Figure 1. Therefore, the proof of Theorem 3 is completed. 
Algorithm 4. Construction of a 4-connected 4-regular locally connected clawfree graph $G$ on $n \geq 7$ vertices.

1. Pick up any vertex $v$.

2. Find 4 neighbors of $v$. Let $v_{2} v_{1} u_{1} u_{2}$ is a hamiltonian path in $N(v)$ and $W_{1}=\left\{v_{1}, v_{2}\right\}$ and $W=\left\{v, u_{1}, u_{2}\right\} \cup W_{1}$, and set $i=1$.

3. Find a neighbor $v_{i+2}$ of $v_{i}$. Set $W_{1}=\left(W_{1} \cup\left\{v_{i+2}\right\}\right)-\left\{v_{i}\right\}$ and $W=W \cup\left\{v_{i}\right\}$

4. If $|W|=|V(G)|$, go to Step 5. Otherwise, set $i=i+1$ and go to 3 .

5 . Check whether $u_{2}$ is adjacent to all vertices of $W_{1}$ and $u_{1}$ is only adjacent to $v_{n-3}$.

We now analyze time in Algorithm 4. Note that $n$ is the number of vertices.

Step 1 needs $O(1)$. Step 2 needs $O(n)$. Step 3 needs $O(n)$ and Step 4 needs $O(1)$. By Theorem 3, such a graph is 4-connected 4-regular locally connected claw-free. Thus we obtain a linear polynomial time algorithm to construct a 4-connected 4-regular locally connected claw-free graph.

\section{References}

1. J. A. Bondy and U.S.R. Murty, Graph Theory with its applications, MacMillan, New York, 1976

2. M.R. Garey and D.S. Johnson, Computers and Intractability-A Guide to the theory of NP-completeness, Freeman, New York, 1979.

3. M. R. Garey, D.S. Johnson and R.E. Tarjan, The planar hamiltonian circuit problem is NP-complete, SIAM J. Computing, 5(1976), 704-714.

4. M. Li, D.G. Corneil and E. Mendelsohn, Pancyclicity and NP-completeness in planar graphs, Discrete Applied Math., 98(2000) 219-225.

5. L. G. Valiant, The complexity of computing the permanent, Theor. Computer Sci., 8(1979), 189-201. 